\title{
Epistasis between intracellular cholesterol trafficking-related genes (NPC1 and ABCA1) and Alzheimer's disease risk
}

Eloy Rodríguez-Rodríguez ${ }^{\mathrm{a}}$, José Luis Vázquez-Higuera ${ }^{\mathrm{a}}$, Pascual Sánchez-Juan ${ }^{\mathrm{a}}$, Ignacio Mateo $^{\mathrm{a}}$, Ana Pozueta ${ }^{\mathrm{a}}$, Ana Martínez-García ${ }^{\mathrm{b}}$, Ana Frank ${ }^{\mathrm{c}}$, Fernando Valdivieso ${ }^{\mathrm{b}}$, José Berciano ${ }^{\mathrm{a}}$, María J. Bullido ${ }^{\mathrm{b}}$, Onofre Combarros ${ }^{\mathrm{a}, *}$

${ }^{a}$ Neurology Service and CIBERNED, “Marqués de Valdecilla” University Hospital (University of Cantabria), Santander, Spain

${ }^{\mathrm{b}}$ Molecular Biology Department and CIBERNED, Centro de Biología Molecular Severo Ochoa (C.S.I.C.-U.A.M.), Madrid, Spain

${ }^{\mathrm{c}}$ Neurology Service and CIBERNED, Hospital Universitario La Paz (U.A.M.), Madrid, Spain

*Corresponding author: Onofre Combarros, Neurology Service, Marqués de Valdecilla University Hospital, Avda. Valdecilla s/n, 39008 Santander, Spain. Tel.: +34 942 202507; fax: +34 942 202655; E-mail: combarro@unican.es 


\section{Abstract}

Aberrant cholesterol metabolism has been implicated in Alzheimer's disease (AD). Recent findings have suggested an interaction of Niemann-Pick C1 (NPC1) and ATP-binding cassette transporter A1 (ABCA1) proteins in intracellular cholesterol transport and in maintaining cell cholesterol balance. Underexpression of NPC1 in concert with underexpression of ABCA1 would result in increased cholesterol accumulation and increased AD risk. We examined a functional polymorphism in the ABCA1 promoter region (-477, rs2422493), and four NPC1 polymorphisms in exon 6 (rs18050810), intron 20 (rs4800488), intron 22 (rs2236707), and intron 24 (rs2510344) capturing 85\% of genetic variability in Hap Map CEU population, in a group of 631 Spanish AD patients and 731 controls. Subjects carrying both the ABCA1 (-477) TT genotype and the NPC1 (exon 6) GG genotype (OR = 1.89; 95\% CI 1.04-3.41), NPC1 (intron 20) AA genotype (OR = 2.05; 95\% CI 1.26-3.33), NPC1 (intron 22) AA genotype (OR = 2.05; 95\% CI 1.18-3.58), or NPC1 (intron 24) GG genotype (OR = 1.89; 95\% CI 1.16-3.07) had a higher risk of developing $\mathrm{AD}$ than subjects without these risk genotypes. Testing for epistatic interaction between genes in the pathway of cholesterol metabolism might be useful for predicting AD risk.

Keywords: Alzheimer's disease; NPC1; ABCA1; Cholesterol; Polymorphism; Epistasis 


\section{Introduction}

Aberrant cholesterol metabolism has been implicated in Alzheimer's disease (AD), with increased cellular cholesterol levels inducing high amyloid beta (A $\beta$ ) production and the development of neurofibrillary tangles [1, 2], which are central to the pathogenesis of AD. Niemann-Pick C1 (NPC1) protein and ATP-binding cassette transporter A1 (ABCA1) protein affect redistributing late endosomal/lysosomal intracellular cholesterol transport to other cellular sites including the plasma membrane and the endoplasmic reticulum [3, 4]. The most striking biochemical feature of NPC1- or ABCA1-deficient cells is an excessive storage of unesterified cholesterol in late endosomes/lysosomes [1, 2]. In recent genome-wide association studies [5, 6], genetic markers of the ABCA1 region showed a weak association to $\mathrm{AD}$ [5], but markers of the NPC1 region were not found associated to AD [5, 6]. In addition, variability in NPC1 [7] and ABCA1 [8-12] genes has been proposed to modify the risk of AD through an independent effect. However, the interactive effect between polymorphisms of two genes (epistasis) may exist without a significant main effect of either of them, and in such cases, the effect would have been missed if polymorphisms of both genes had not been tested jointly [13]. Therefore, and considering the postulated common pathway of NPC1 and ABCA1 in intracellular cholesterol trafficking, we examined the combined contribution of these genes to the susceptibility for AD in a case-control study. Our hypothesis is that there may be functional variants in the NPC1 and ABCA1 genes that would predispose people to AD due to underexpression of the NPC1 gene in concert with underexpression of the ABCA1 gene. We focused on a functional polymorphism in the ABCA1 (-477, rs2422493) gene [14] previously associated with AD risk [11, 15], and we also examined one NPC1 polymorphism in exon 6 (rs18050810) previously analyzed in AD [7] and three other NPC1 polymorphisms in intron 20 (rs4800488), intron 22 (rs2236707), and intron 24 (rs2510344).

\section{Methods}


Subjects. The study included 631 AD patients (65\% women; mean age at study 75.9 years; SD 8.1; range 61-109 years; mean age at onset 72.8 years; SD 7.8; range 60-100 years) who met NINCDS/ADRDA criteria for probable AD [16]. All AD cases were defined as sporadic because their family history did not mention any first-degree relative with dementia. AD patients were recruited from the Departments of Neurology of University Hospital “Marqués de Valdecilla” (Santander, Spain), and Hospital “La Paz” (Madrid, Spain). The large majority of patients were living in the community and had been referred by their general practitioner; few had been admitted from hospital wards or nursing home facilities. Control subjects were 731 unrelated individuals (65\% women; mean age 78.6 years; SD 9.4; range 60-104 years) randomly selected from nursing homes. These subjects had complete neurologic and medical examinations that showed that they were free of significant illness and had Mini Mental State Examination scores of 28 or more, which were verified by at least one subsequent annual following-up assessment. The controls arose from the same base population as the cases. The AD and control samples were Caucasians originating from a limited geographical area in northern Spain (Santander) and from the central area of Spain (Madrid).

Genotyping. Blood samples were taken after written informed consent had been obtained from the subjects or their representatives. The study was approved by the ethical committees of the University Hospital “Marqués de Valdecilla” and the Hospital “La Paz”. Genotyping of NPC1 (rs18050810, rs4800488, rs2236707, and rs2510344) polymorphism was performed by a TaqMan single-nucleotide-polymorphism assay (Applied Biosystems, Warrington, Cheshire, UK) and an ABI PRISM 7000 or 7900HT sequence detection systems (Applied Biosystems). We used data from the HapMap project (http://www.hapmap.org) to select the 4 htSNPs capturing 85\% of NPC1 genetic variability in Caucasians. SNPs were chosen among those with minor allele frequencies $\geq 5 \%$ using Haploview v3.2 software (http://www.broad.mit.edu/mpg/haploview) with an $\mathrm{r}^{2}$ threshold of 0.8. The ABCA1 (-477, rs2422493) polymorphism was determined as described previously [9]. APOE genotyping was 
performed by amplification of the 4th exon of the APOE gene by PCR with biotinylated primers, followed by reverse hybridization on nitrocellulose strips, using the INNO-LIPA ApoE assay (Innogenetics NV, Ghent, Belgium), or by HhaI restriction analysis.

Statistical analysis. Hardy-Weinberg equilibrium (HWE) was calculated in the control population using Pearson's $\chi^{2}$ statistics. We assessed pairwise linkage disequilibrium (LD) between the 4 NPC1 htSNPs by $D^{\prime}$ and $r^{2}$ statistics. Haplotype reconstruction and their frequencies in cases and controls were estimated by an expectation-maximization algorithm, method implemented in Haploview 3.32. Pearson's $\chi^{2}$ statistics were performed to compare genotype, allele, and haplotype distribution of the patients and control for each SNP. Genotypic and allelic distributions were assessed by logistic regression using SPSS software. Odds ratios (ORs) and $p$-values adjusted by age, gender and APOE status were also calculated by logistic regression. All statistical analyses were performed with the package SPSS 13.0 for Windows (SPSS, Inc, Chicago, Illinois).

\section{Results}

Control groups for ABCA1 $(-477)(p=0.57)$, NPC1 (exon 6) $(p=0.60)$, NPC1 (intron 20) $(p=$ 0.99), NPC1 (intron 22) ( $\mathrm{p}=0.80)$, and NPC1 (intron 24) $(\mathrm{p}=0.75)$ polymorphisms were within the range of Hardy-Weinberg equilibrium. In our Spanish population the four NPC1 polymorphisms were in strong linkage disequilibrium: exon 6 and intron 20, $\mathrm{D}^{\prime}=0.99$; exon 6 and intron 22, $\mathrm{D}^{\prime}=0.96$; exon 6 and intron 24, $\mathrm{D}^{\prime}=0.95$; intron 20 and intron 22, $\mathrm{D}^{\prime}=0.95$; intron 20 and intron 24, $\mathrm{D}^{\prime}=0.96$; intron 22 and intron 24, $\mathrm{D}^{\prime}=0.92$. When the risk was considered for a single polymorphism, the distribution of the allele and genotype frequencies of the ABCA1 (-477), NPC1 (exon 6), NPC1 (intron 20), NPC1 (intron 22), and NPC1 (intron 24) polymorphisms did not differ significantly between $\mathrm{AD}$ and control groups (Table 1). The overall frequencies of the NPC1 haplotypes were not statistically different between cases and controls (data not shown). However, the subjects carrying both the ABCA1 (-477) TT genotype 
and the NPC1 (exon 6) GG genotype (OR = 1.89; 95\% CI 1.04-3.41; p = 0.034), NPC1 (intron 20) AA genotype (OR = 2.05; 95\% CI 1.26-3.33; $\mathrm{p}=0.004)$, NPC1 (intron 22) AA genotype $(\mathrm{OR}=2.05 ; 95 \% \mathrm{CI} 1.18-3.58 ; \mathrm{p}=0.01)$, or NPC1 (intron 24) GG genotype $(\mathrm{OR}=1.89 ; 95 \%$ CI 1.16-3.07; $p=0.01$ ) had a higher risk of developing AD than subjects without these risk genotypes, suggesting a gene-gene interaction (Table 2). Since the four NPC1 polymorphisms in our study are in almost complete linkage disequilibrium forming one block, we have tested only three independent comparisons examining the interaction between the ABCA1 and NPC1 genes; therefore, when applying the conservative Bonferroni adjustment, statistical significance should be set up at $\mathrm{p}<0.017$, which is reached by the interaction between ABCA1 (-477) and NPC1 (intron 20) $(p=0.004)$, NPC1 intron $22(p=0.01)$, or NPC1 (intron 24) $(p=0.01)$.

\section{Discussion}

We have shown, for the first time, that the NPC1 and ABCA1 genes may interact in determining the risk for $\mathrm{AD}$, with subjects carrying both the ABCA1 (-477) TT genotype and the NPC1 (exon 6) GG genotype, NPC1 (intron 20) AA genotype, NPC1 (intron 22) AA genotype, or NPC1 (intron 24) GG genotype having a higher risk of developing AD. De novo synthesis in glial cells is responsible for practically all cholesterol present in the brain, and once synthesized, astrocytes secrete the cholesterol predominantly via the cholesterol transporter ABCA1, which reduces cellular cholesterol and inhibits $A \beta$ production [17]. The deficiency of ABCA1 results in accumulation of cholesterol in cultured glia and elevated $A \beta$ levels in the brain of transgenic mouse models of $\mathrm{AD}[18,19]$. Genetic variants in ABCA1 have been proposed to modify the risk of $\mathrm{AD}$ [8-12], and homozygosity for the ABCA1 (-477) $\mathrm{T}$ allele in concert with homozygosity for the HMGCR 5'-UTR (the major rate-limiting enzyme in the novo synthesis of cholesterol,) G allele has been associated with increased AD risk [15]. The ABCA1 (-477) polymorphism located in the promoter region has a functional effect: luciferase reporter assay indicates that the ABCA1 (-477) C allele has significantly higher promoter activity than the 
ABCA1 (-477) T allele promoter [14]. So, we hypothesized that subjects carrying the low ABCA1 (-477) producer TT genotype might show a higher AD risk due to their genetic inability to induce a more effective ABCA1 protective response.

The present study indicates that an AD risk effect of ABCA1 (-477) producer TT genotype is only apparent in the presence of NPC1 (exon 6) GG genotype, NPC1 (intron 20) AA genotype, NPC1 (intron 22) AA genotype, or NPC1 (intron 24) GG genotype. In a preliminary study conducted in the Polish population, there were gradients of two non-synonymous (rs18050810 in exon 6 and rs1788799 in exon 12) SNP's allele frequencies in NPC1 gene from centenarians through normal controls to sporadic late-onset AD, indicating a role for NPC1 gene in AD and/or aging [7]. In addition to this SNP rs18050810 (exon 6) of the original paper, we genotyped other SNPs in introns 20, 22 and 24 capturing 85\% of NPC1 genetic variability in CEU Hap Map population; in our sample, all the SNPs analyzed were located in a single haplotype block in almost complete linkage disequilibrium. Although there are no relevant functional assays of any of these NPC1 studied polymorphisms, the most likely mechanism of the association of the NPC1 genetic variants with AD is the loss of function of NPC1. The NPC1 is an intracellular chaperone involved in the transport of cholesterol from late endosomes/lysosomes to the endoplasmic reticulum and the plasma membrane [3]. NPC disease is a progressive neurological disorder in which large amounts of cholesterol accumulate in late endosomes/lsysosomes in NPC1-deficient cells, caused by mutations in the NPC1 gene [2]. Neurofibrillary tangles have been detected in brains of juvenile and adult cases of NPC disease, these tangles being immunocytochemically and morphologically indistinguishable from the tangles in AD brains and show the same pattern of tau hyperphosphorylation [20]. In addition, tangle-bearing neurons in $\mathrm{AD}$ contain more unesterified cholesterol than do tangle-free neurons [21] suggesting that a sequestration of cholesterol, such as occurs in NPC disease, might influence tangle formation. It has been suggested that cholesterol may also modulate $A \beta$ formation, a causative factor of $A D$ [1]; so, the intracellular accumulation of cholesterol in the brain of a mouse model of NPC 
disease enhances amyloidogenesis via enhancement of $\gamma$-secretase activity, which correlates with an increase in $A \beta$ levels [22]. Recent findings have suggested an interaction of ABCA1 and NPC1 proteins in intracellular cholesterol trafficking: in cultured NPC1-deficient fibroblasts ABCA1 expression was decreased and the ABCA1-dependent efflux of cholesterol was impaired [23].

Although information about the expression of ABCA1 (-477), NPC1 (exon 6), NPC1 (intron 20), NPC1 (intron 22), and NPC1 (intron 24) at the brain level in subjects with different genotypes is lacking, it is tempting to speculate that a reduction in the expression of ABCA1 and NPC1 in carriers of both the ABCA1 (-477) TT genotype and the NPC1 (exon 6) GG genotype, NPC1 (intron 20) AA genotype, NPC1 (intron 22) AA genotype, or NPC1 (intron 24) GG genotype would lead to higher cholesterol levels in neurons and glial cells, which in turn could induce $A \beta$ production and tangle formation and increased $A D$ risk. Our sample size had $90 \%$ power to detect an OR of 2.0 or higher for an effect of ABCA1 (-477) TT genotype and NPC1 (intron 20) AA genotype on $\mathrm{AD}$ risk. It is noticeable that no association with $\mathrm{AD}$ risk was obtained from the examination of the main effects of ABCA1 and NPC1 independently, but an important result has been derived from the study of their epistatic (gene-gene) interaction [13]. In fact, with the current one-SNP-at-a-time approach to genomewide association studies, failure to replicate a genetic association in a second sample can be an indication that single SNPs contribute to disease susceptibility through interactions with one or more other SNPs [24]. Moreover, it has been proposed that patterns of SNPs in biochemical pathways, such as the cholesterol trafficking pathway analyzed in the present study, are more likely to replicate than individual and non-related SNPs [25]. Additional studies using different sets of patients and control subjects are required to verify the validity of our hypotheses (genetic and biologic).

\section{Conflicts of interest: none}


Acknowledgements: C. Sánchez-Quintana was involved in the DNA sample collections and genotyping analysis from Santander. We thank Drs. P. Gil and P. Coria for their cooperation in the generation of the case-control samples from Madrid, and to I. Sastre for the maintenance and control of the DNA bank (Madrid). This work was made possible by the generous participation of the patients, the control subjects, and their families. This study was supported by grants from FIS (PI080139) and CIBERNED (CB06/07/0037).

\section{References}

[1] Vance JE, Hayashi H, Karten B. (2005) Cholesterol homeostasis in neurons and glial cells. Semin Cell Dev Biol. 16, 193-212.

[2] Karten B, Peake KB, Vance JE. (2009) Mechanisms and consequences of impaired lipid trafficking in Niemann-Pick type C1-deficient mammalian cells. Biochim Biophys Acta 1791, 659-670.

[3] Neufeld EB, Wastney M, Patel S, Suresh S, Cooney AM, Dwyer NK, Roff CF, Ohno K, Morris JA, Carstea ED, Incardona JP, Strauss III JF, Vanier MT, Patterson MC, Brady RO, Pentchev PG, Blanchette-Mackie EJ. (1999) The Niemann-Pick C1 protein resides in a vesicular compartment linked to retrograde transport of multiple lysosomal cargo. J Biol Chem. 274, 96279635.

[4] Neufeld EB, Stonik JA, Demosky Jr SJ, Knapper CL, Combs CA, Cooney A, Comly M, Dwyer N, Blanchette-Mackie J, Remaley AT, Santamarina-Fojo S, Brewer HB. (2004) The ABCA1 transporter modulates late endocytic trafficking: insights from the correction of the genetic defect in Tangier disease. J Biol Chem. 279, 15571-15578.

[5] Harold D, Abraham R, Hollingworth P, Sims R, Gerrish A, Hamshere ML, Pahwa JS, Moskvina V, Dowzell K, Williams A, Jones N, Thomas C, Stretton A, Morgan A.R, Lovestone S, Powell J, Proitsi P, Lupton MK, Brayne C, Rubinsztein DC, Gill M, Lawlor B, Lynch A, Morgan K, Brown KS, Passmore PA, Craig D, McGuinness B, Todd S, Holmes C, Mann D, 
Smith AD, Love S, Kehoe PG, Hardy J, Mead S, Fox N, Rossor M, Collinge J, Maier W, Jessen F, Schürmann B, van den Bussche H, Heuser I, Kornhuber J, Wiltfang J, Dichgans M, Frölich L, Hampel H, Hüll M, Rujescu D, Goate AM, Kauwe JS, Cruchaga C, Nowotny P, Morris JC, Mayo K, Sleegers K, Bettens K, Engelborghs S, De Deyn PP, Van Broeckhoven C, Livingston G, Bass NJ, Gurling H, McQuillin A, Gwilliam R, Deloukas P, Al-Chalabi A, Shaw CE, Tsolaki M, Singleton AB, Guerreiro R, Mühleisen TW, Nöthen MM, Moebus S, Jöckel KH, Klopp N, Wichmann HE, Carrasquillo MM, Pankratz VS, Younkin SG, Holmans PA, O'Donovan M, Owen MJ, Williams J. (2009) Genome-wide association study identifies variants at CLU and PICALM associated with Alzheimer's disease. Nat Genet. 41, 1088-1093.

[6] Lambert JC, Heath S, Even G, Campion D, Sleegers K, Hiltunen M, Combarros O, Zelenika D, Bullido MJ, Tavernier B, Letenneur L, Bettens K, Berr C, Pasquier F, Fiévet N, Barberger-Gateau P, Engelborghs S, De Deyn P, Mateo I, Franck A, Helisalmi S, Porcellini E, Hanon O, European Alzheimer's Disease Initiative Investigators, de Pancorbo MM, Lendon C, Dufouil C, Jaillard C, Leveillard T, Alvarez V, Bosco P, Mancuso M, Panza F, Nacmias B, Bossù P, Piccardi P, Annoni G, Seripa D, Galimberti D, Hannequin D, Licastro F, Soininen H, Ritchie K, Blanché H, Dartigues JF, Tzourio C, Gut I, Van Broeckhoven C, Alpérovitch A, Lathrop M, Amouyel P. (2009) Genome-wide association study identifies variants at CLU and CR1 associated with Alzheimer’s disease. Nat Genet. 41, 1094-1099.

[7] Erickson RP, Larson-Thomé K, Weberg L, Szybinska A, Mossakowska M, Styczynska M, Barcikowska M, Kuznicki J. (2008) Variation in NPC1, the gene encoding Niemann-Pick C1, a protein involved in intracellular cholesterol transport, is associated with Alzheimer disease and/or aging in the Polish population. Neurosci Lett. 447, 153-157.

[8] Katzov H, Chalmers K, Palmgren J, Andreasen N, Johansson B, Cairns NJ, Gatz M, Wilcock GK, Love S, Pedersen NL, Brookes AJ, Blennow K, Kehoe PG, Prince JA. (2004) Genetic variants of ABCA1 modify Alzheimer disease risk and quantitative traits related to $\beta$ amyloid metabolism. Hum Mutat. 23, 358-367. 
[9] Rodríguez-Rodríguez E, Mateo I, Llorca J, Sánchez-Quintana C, Infante J, GarcíaGorostiaga I, Sánchez-Juan P, Berciano J, Combarros O. (2007) Association of genetic variants of ABCA1 with Alzheimer's disease risk. Am J Med Genet. 144B, 964-968.

[10] Sundar PD, Feingold E, Minster RL, DeKosky ST, Kamboh MI. (2007) Gender-specific association of ATP-binding cassette transporter 1 (ABCA1) polymorphisms with the risk of lateonset Alzheimer's disease. Neurobiol Aging 28, 856-862.

[11] Wavrant-De Vrièze F, Compton D, Womick M, Arepalli S, Adighibe O, Li P, Pérez-Tur J, Hardy J. (2007) ABCA1 polymorphisms and Alzheimer's disease. Neurosci Lett. 416, 180183.

[12] Reynolds CA, Hong MG, Eriksson UK, Blennow K, Bennet AM, Johansson B, Malmberg B, Berg S, Wiklund F, Gatz M, Pedersen NL, Prince JA. (2009) A survey of ABCA1 sequence variation confirms association with dementia. Hum Mutat. 30, 1348-1354.

[13] Combarros O, Cortina-Borja M, Smith AD, Lehmann DJ. (2009) Epistasis in sporadic Alzheimer's disease. Neurobiol Aging 30, 1333-1349.

[14] Kyriakou T, Hodgkinson C, Pontefract DE, Iyengar S, Howell WM, Wong Y, Eriksson P, Ye S. (2005) Genotypic effect of the $-565 C>T$ polymorphism in the ABCA1 gene promoter on ABCA1 expression and severity of atherosclerosis. Arterioscler Thromb Vasc Biol. 25, 418-423.

[15] Rodríguez-Rodríguez E, Mateo I, Infante J, Llorca J, García-Gorostiaga I, VázquezHiguera JL, Sánchez-Juan P, Berciano J, Combarros O. (2009) Interaction between HMGCR and ABCA1 cholesterol-related genes modulates Alzheimer's disease risk. Brain Res. 1280, 166171.

[16] McKhann G, Drachman D, Folstein M, Katzman R, Price D, Stadlan EM. (1984) Clinical diagnosis of Alzheimer disease: Report of the NINCDS-ADRDA Work Group under the auspices of the Department of Health and Human Services Task Force on Alzheimer Disease. Neurology 24, 939-944. 
[17] Dietschy JM, Turley SD. (2001) Cholesterol metabolism in the brain. Curr Opin Lipidol. 12, 105-112.

[18] Koldamova R, Staufenbiel M, Lefterov I. (2005) Lack of ABCA1 considerably decreases brain ApoE level and increases amyloid deposition in APP23 mice. J Biol Chem. 280, 4322443235.

[19] Wahrle SE, Jiang H, Parsadanian M, Hartman RE, Bales KR, Paul SM, Holtzman DM. (2005) Deletion of Abca1 increases A $\beta$ deposition in the PDAPP transgenic mouse model of Alzheimer disease. J Biol Chem. 280, 43236-43242.

[20] Distl R, Treiber-Held S, Albert F, Meske V, Harzer K, Ohm TG. (2003) Cholesterol storage and tau pathology in Niemann-Pick type C disease in the brain. J Pathol. 200,104-111.

[21] Distl R, Meske V, Ohm TG. (2001) Tangle-bearing neurons contain more free cholesterol than adjacent tangle-free neurons. Acta Neuropathol. 101, 547-554.

[22] Burns M, Gaynor K, Olm V, Mercken M, LaFrancois J, Wang L, Mathews PM, Noble W, Matsuoka Y, Duff K. (2003) Presenilin redistribution associated with aberrant cholesterol transport enhances $\beta$-amyloid production in vivo. J Neurosci. 23, 5645-5649.

[23] Choi HY, Karten B, Chan T, Vance JE, Greer WL, Heidenreich RA, Garver WS, Francis GA. (2003) Impaired ABCA1-dependent lipid efflux and hypoalphalipoproteinemia in human Niemann-Pick type C disease. J Biol Chem. 278, 32569-32577.

[24] Moore JH, Williams SM. (2009) Epistasis and its implication for personal genetics. Am J Hum Genet. 85, 309-320.

[25] Wang K, Li M, Bucan M. (2007) Pathway-based approaches for analysis of genomewide association studies. Am J Hum Genet. 81, 1278-1283. 
Table 1. Distribution of ABCA1 and NPC1 polymorphisms in patients and controls

\begin{tabular}{lcc}
\hline Polymorphism & Patients & Controls \\
\hline ABCA1 (-477) (rs2422493) & & \\
CC & $180(0.29)$ & $197(0.27)$ \\
CT & $285(0.45)$ & $372(0.51)$ \\
TT & $166(0.26)$ & $162(0.22)$ \\
Total & 631 & 731 \\
Allele frequency C/T & $0.51 / 0.49$ & $0.52 / 0.48$ \\
NPC1 (exon 6) (rs18050810) & $259(0.42)$ & \\
AA & $278(0.45)$ & $300(0.42)$ \\
AG & $79(0.13)$ & $327(0.45)$ \\
GG & 616 & $97(0.13)$ \\
Total & $0.65 / 0.35$ & 724 \\
Allele frequency A/G & & $0.64 / 0.36$ \\
NPC1 (intron 20) (rs4800488) & $197(0.32)$ & \\
CC & $300(0.48)$ & $222(0.31)$ \\
CA & $125(0.20)$ & $359(0.49)$ \\
AA & 622 & $145(0.20)$ \\
Total & $0.56 / 0.44$ & 726 \\
Allele frequency C/A & & $0.55 / 0.45$ \\
NPC1 (intron 22) (rs2236707) & $230(0.37)$ & $269(0.37)$ \\
GG & $293(0.48)$ & $349(0.48)$ \\
GA & $93(0.15)$ & $109(0.15)$ \\
AA & 616 & 727 \\
Total & $0.61 / 0.39$ & $0.61 / 0.39$ \\
Allele frequency G/A & & \\
NPC1 (intron 24) (rs2510344) & $201(0.33)$ & $218(0.30)$ \\
AA & $293(0.47)$ & $364(0.50)$ \\
AG & $124(0.20)$ & $145(0.20)$ \\
GG & 618 & $0.55 / 0.45$ \\
Total & $0.56 / 0.44$ & \\
Allele frequency A/G & & \\
\hline & & \\
\hline & & \\
\hline
\end{tabular}

Figures in parentheses indicate frequencies 
Table 2. Odds ratios for Alzheimer's disease risk according to interaction between ABCA1 genotypes with NPC1 polymorphisms

\begin{tabular}{|c|c|c|c|c|c|c|c|}
\hline NPC1 genotypes & $\begin{array}{l}\text { ABCA1 (-477) } \\
\text { TT genotype }\end{array}$ & AD cases & Controls & OR $(95 \% \mathrm{CI}) *$ & $\mathrm{p}^{*}$ & OR $(95 \% \mathrm{CI}) * *$ & $\mathrm{p} * *$ \\
\hline \multicolumn{8}{|l|}{ NPC1 (exon 6) GG genotype } \\
\hline- & - & 405 & 485 & 1 (Reference) & & 1 (Reference) & \\
\hline- & + & 132 & 142 & $1.13(0.84-1.46)$ & 0.43 & $1.07(0.78-1.45)$ & 0.67 \\
\hline+ & - & 49 & 78 & $0.75(0.51-1.10)$ & 0.14 & $0.80(0.52-1.23)$ & 0.31 \\
\hline+ & + & 30 & 19 & $1.89(1.04-3.41)$ & 0.034 & $1.83(0.95-3.52)$ & 0.068 \\
\hline \multicolumn{8}{|c|}{ NPC1 (intron 20) AA genotype } \\
\hline- & - & 383 & 450 & 1 (Reference) & & 1 (Reference) & \\
\hline- & + & 114 & 131 & $1.02(0.76-1.36)$ & 0.87 & $1.01(0.73-1.39)$ & 0.95 \\
\hline+ & - & 76 & 117 & $0.76(0.55-1.05)$ & 0.10 & $0.85(0.59-1.22)$ & 0.38 \\
\hline+ & + & 49 & 28 & $2.05(1.26-3.33)$ & 0.004 & $1.85(1.07-3.18)$ & 0.026 \\
\hline \multicolumn{8}{|c|}{ NPC1 (intron 22) AA genotype } \\
\hline- & - & 399 & 479 & 1 (Reference) & & 1 (Reference) & \\
\hline- & + & 124 & 139 & $1.07(0.81-1.41)$ & 0.62 & $1.04(0.76-1.42)$ & 0.78 \\
\hline+ & - & 57 & 88 & $0.77(0.54-1.11)$ & 0.16 & $0.86(0.58-1.29)$ & 0.58 \\
\hline+ & + & 36 & 21 & $2.05(1.18-3.58)$ & 0.01 & $2.00(1.07-3.74)$ & 0.028 \\
\hline \multicolumn{8}{|c|}{ NPC1 (intron 24) GG genotype } \\
\hline- & - & 377 & 450 & 1 (Reference) & & 1 (Reference) & \\
\hline- & + & 117 & 132 & $1.05(0.79-1.40)$ & 0.69 & $1.05(0.76-1.45)$ & 0.73 \\
\hline+ & - & 78 & 116 & $0.80(0.58-1.10)$ & 0.17 & $0.89(0.62-1.27)$ & 0.52 \\
\hline+ & + & 46 & 29 & $1.89(1.16-3.07)$ & 0.01 & $1.71(0.99-2.94)$ & 0.051 \\
\hline
\end{tabular}

ABCA1 (-477) TT (-) =CC+CT; NPC1 (exon 6) GG (-) = AA+AG; NPC1 (intron 20) AA (-) = CC+CA; NPC1 (intron 22) AA (-) = GG+GA; NPC1 (intron 24) GG (-) = AA+AG; * crude odds ratios; ** odds ratios adjusting by age, sex and APOE genotype (using multiple logistic regression) 